\title{
GLOBAL COORDINATES OF THE SNS ACCELERATOR COMPLEX*
}

\author{
W. Wan, J. Galambos and J. Wei, ORNL, Oak Ridge, TN 37831, USA
}

\begin{abstract}
The global coordinates of the Spallation Neutron Source are determined following the officially approved site wide coordinate system. Lattice output files from computer codes such as PARMILA, TRANSPORT and MAD are used. Integration of various parts of the facility is critical because they are designed by people from different partner labs. Issues related to integration, including interfacing between linac and ring, between beam transport line and the target and between accelerator and the conventional facility are presented.
\end{abstract}

\section{INTRODUCTION}

The Spallation Neutron Source (SNS) Project is currently under construction with the participation of six different national labs [1]. The fact that work is being carried out at various locations simultaneously poses new challenge to coordination and information flow. The establishment of the global coordinate system of the accelerator complex deals with every component of the complex and directly involves people from four of the six labs. In order to ensure project-wide consistency, the origin and the axes were agreed upon and defined in the form of an official document [2]. Then interfaces of different parts were clarified and the global coordinates of a few key points were determined. Finally, the coordinates of all dipoles and quadrupoles were calculated. Specifically, the global coordinate system is first outlined, followed by a section describing the interfaces and the critical issues encountered. The last section contains discussions on present status and future plan.

\section{SNS GLOBAL COORDINATE SYSTEM}

The current SNS global coordinate system was proposed by the SNS Survey \& Alignment Group and adopted by the whole project [2]. It is illustrated in Figure 1, which is a two-dimensional drawing of the SNS site. The origin of $\mathrm{Y}$-axis lies in the plane of the accumulator ring. To avoid unnecessary confusion, the coordinates of the origin are defined as $(20000,10000,2000)$ in the order of $(Z, X, Y)$.

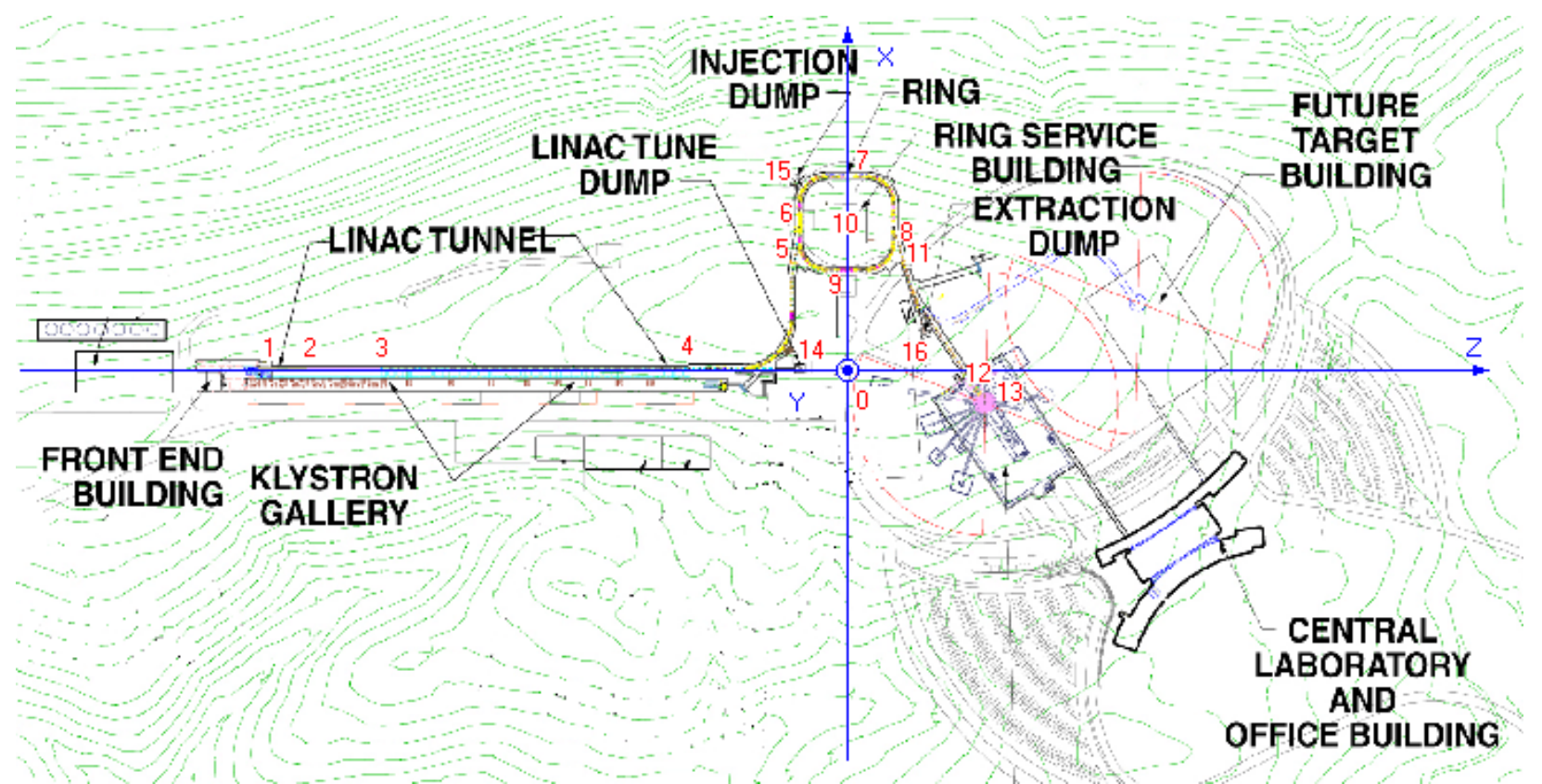

Figure 1: SNS site map with key points marked

\section{INTERFACES AND INTEGRATION}

The issue of interfaces becomes a critical one due to the fact that different parts of the SNS facility was designed by different people at different labs using different design codes. The front end was designed and built by a team at

*Work supported by the U.S. Department of Energy under contract No. DE-AC05-96OR22464.
Lawrence Berkeley Lab, the linac by a team at Los Alamos National Lab, the accumulator ring and the beam transfer lines by people at Brookhaven National Lab; and the target station was by people at Oak Ridge National Lab. The linac was designed using PARMILA [3], the beam transfer lines using TRANSPORT [4] and the ring using MAD [5]. An Excel spreadsheet was developed to integrate the various parts into a coherent whole. In order to build the spreadsheet, the geometrical relations among 
the parts have to be known. Four points are essential for the integration, which are the beginning of the High Enegy Beam Transport (HEBT), the end of the HEBT (beginning of the Injection dump line), the beginning of the Ring to Target Beam Transport (RTBT) and the end of the RTBT.

The interface point between the linac and the HEBT is defined in a straightforward way, which is the center of the magnet named HEBT.QV1. The end of the PARMILA output of the linac is the beginning of the TRANSPORT output of the HEBT. The HEBT ends at the stripping foil, where the $\mathrm{H}^{-}$ions are converted into protons. Due to transverse painting [6], the center of the injected beam at the foil does not lie on the center of the straight section of the ring (Figure 2). It is $14 \mathrm{~cm}$ outside and $4.6 \mathrm{~cm}$ above the ring. Specifically, the relative position of this point to the midpoint of the injection straight, in the form of the global coordinate system described above, is $(-0.14,-0.605,0.046)$. The RTBT starts at $3.29 \mathrm{~m}$ upstream of the Lambertson magnet, whose upstream end's relative position to the midpoint of the extraction straight is $(0,-3.4376,-0.1826)$ (Figure 3). The end of the RTBT and the distance between it and the center of the target are also determined. The numbers above were obtained from sources other than the lattice files, which were incorporated into the spreadsheet manually. With the exception of the injection dump line, the global coordinates of all magnets can be determined.

The uniqueness of the injection dump line lies in the fact that there are two beams separated horizontally in the beam pipe. After the foil at the end of the HEBT, most $\mathrm{H}^{-}$ ions are converted into protons. Some are partly stripped and become $\mathrm{H}^{0}$, which traverse magnet INJBND3 with no bending. Others are not stripped and remain $\mathrm{H}^{-}$, which are bent $2.4^{\circ}$ after INJBND3 (Figure 2). All $\mathrm{H}^{0}$ and $\mathrm{H}^{-}$ions are fully stripped by a thick foil in front of INJBND4 and sent to the injection dump. The reference orbit (center of the beam pipe) is defined as the path of a particle that is bend $1.2^{\circ}$ by INJBND3 and behaves as a proton afterwards.

The first result of the work is a list of the coordinates of 16 key points (Table 1), which allows the Survey \& Alignment Group and people in the Conventional Facilities Division to proceed with their work.

\section{STATUS AND PLAN}

Currently, a list of the coordinates of all dipoles and quadrupoles of the ring and the beam transfer lines has been released. That of the linac is due shortly pending the release of the final design. Coordinates of other devices such as sextupoles, correction magnets and BPMs will be provided in the coming months. The data will be incorporated in a comprehensive database currently under development. More work will also be done to increase the degree of automation in calculating the coordinates.

\section{ACKNOWLEDGEMENTS}

The authors would like to thank H. Takeda, J. Negrin, D. Raparia, Y. Papaphilippou, J. Error, D. Stout, C. Peters and T. McManamy for providing important data and stimulating discussions.

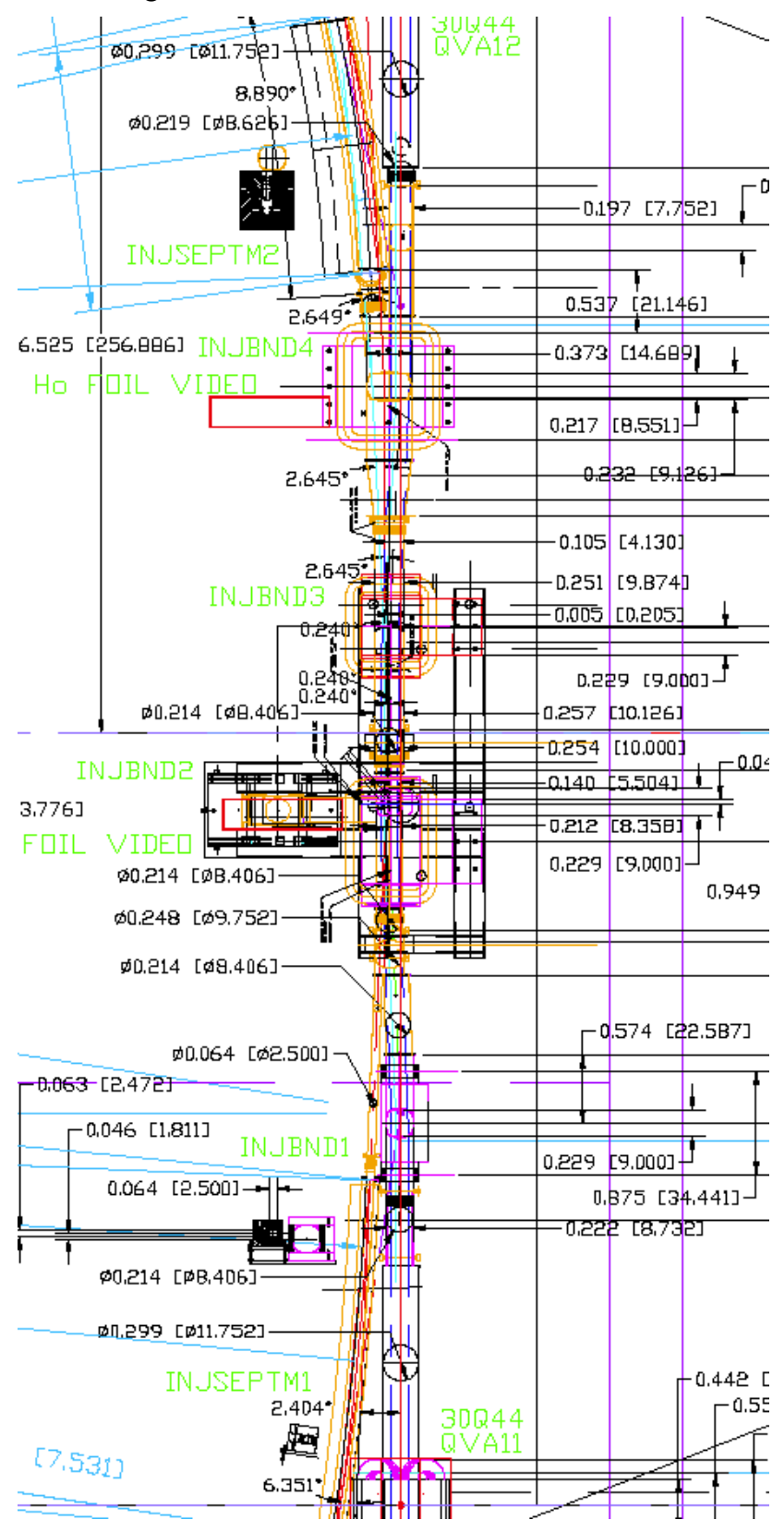

Figure 2: AutoCAD drawing of the injection area

\section{REFERENCES}

[1] T. Mason, these proceedings.

[2] J. Error, private communication.

[3] H. Takeda, LA-UR-98-4478 (2000).

[4] D. C. Carey, K. L. Brown and F. Rothacker, SLAC-R530, Fermilab-Pub-98-310, UC-414 (1998).

[5] H. Grote and F. C. Iselin, CERN-SL-90-13 (AP) (Rev. 5) (1996).

[6] J. Wei et. al, these proceedings. 


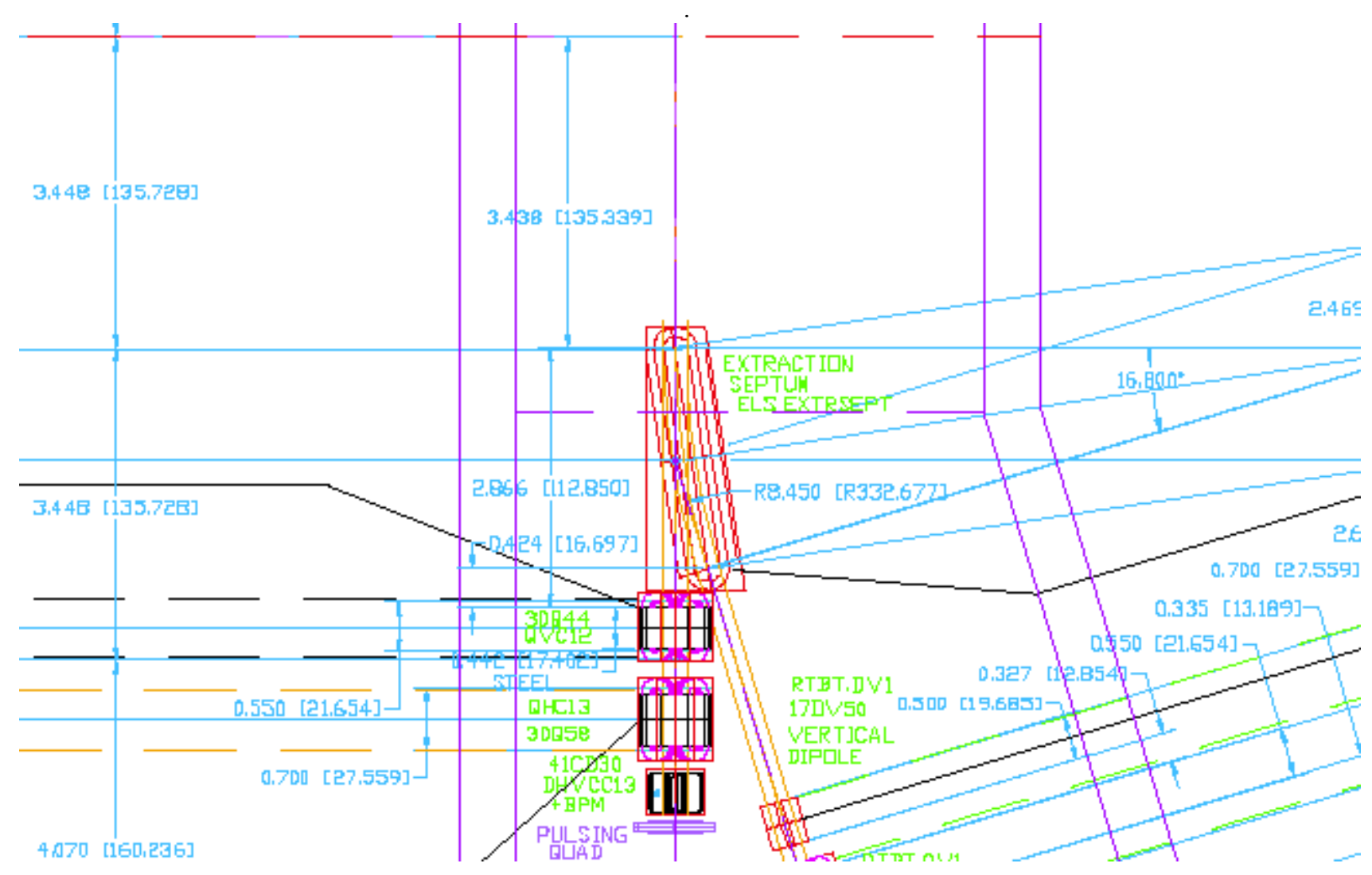

Figure 3: AutoCAD drawing of the extraction area

\begin{tabular}{|l|l|l|c|c|c|}
\hline$\#$ & Name & Location & $\mathrm{Z}$ & $\mathrm{X}$ & $\mathrm{Y}$ \\
\hline 0 & Complex center & Central Monument 00 (MON00) & $20,000.000000$ & $10,000.000000$ & $2,000.000000$ \\
\hline 1 & FE end & Last quad in MEBT (QM14) & $19,549.720844$ & $10,000.000000$ & $2,000.046000$ \\
\hline 2 & DTL end & Quad between DTL and CCL & $19,586.686974$ & $10,000.000000$ & $2,000.046000$ \\
\hline 3 & CCL end & Last quad in CCL-SC Region & $19,643.268831$ & $10,000.000000$ & $2,000.046000$ \\
\hline 4 & HEBT beginning & First quad in HEBT (HEBT.QV1) & $19,881.174844$ & $10,000.000000$ & $2,000.046000$ \\
\hline 5 & HEBT end & Last quad in HEBT (HEBT.QH34) & $19,963.886411$ & $10,102.299981$ & $2,000.046000$ \\
\hline 6 & Ring section A & First quad after foil (QVA12) & $19,964.669100$ & $10,117.136218$ & $2,000.000000$ \\
\hline 7 & Ring section B & QVB12 & $20,006.525000$ & $10,145.942118$ & $2,000.000000$ \\
\hline 8 & Ring section C & QVC12 & $20,035.330900$ & $10,104.086218$ & $2,000.000000$ \\
\hline 9 & Ring section D & QVD12 & $19,993.475000$ & $10,075.280318$ & $2,000.000000$ \\
\hline 10 & Ring center & Center of ring & $20,000.000000$ & $10,110.611218$ & $2,000.000000$ \\
\hline 11 & RTBT beginning & First quad in RTBT (RTBT.QV1) & $20,036.790003$ & $10,101.093419$ & $1,999.771400$ \\
\hline 12 & RTBT end & Last quad in RTBT (RTBT.QH30) & $20,098.564592$ & $9,982.566321$ & $1,999.817400$ \\
\hline 13 & Target center & Center of target & $20,104.489012$ & 9973.648848 & $1,999.817400$ \\
\hline 14 & Linac dump & Center of the linac dump & $19,963.424844$ & $10,000.000000$ & $2,000.046000$ \\
\hline 15 & Injection dump & Center of the injection dump & $19,958.312420$ & $10,142.496311$ & $2,000.046000$ \\
\hline 16 & Extraction dump & Center of the extraction dump & $20,060.548572$ & $10,022.394270$ & $1,999.817400$ \\
\hline
\end{tabular}

Table 1: Coordinates of key points along the reference orbit 